\title{
Earth Science Engagement through Art and Astronaut Photography
}

\author{
Andrea L. Meado ${ }^{1}$, William L. Stefanov ${ }^{2}$, Melissa D. Higgins ${ }^{1}$, Lisa A. Vanderbloemen ${ }^{1}$ \\ ${ }^{1}$ Jacobs, NASA Johnson Space Center, Houston, TX 77058, USA \\ ${ }^{2}$ NASA Johnson Space Center, Houston, TX 77058, USA
}

For over five decades of manned spaceflight missions, NASA astronauts have taken extraordinary photographs of Earth's surface and dynamic processes. Humans on board the International Space Station (ISS) have a unique platform to perform Earth observations at various viewing angles, seasons, and times of day. Astronaut photos taken from the ISS comprise a true-color (RGB) dataset taken with multiple handheld digital cameras and lens types (prior to 2004, film cameras were in use). Earth observations through astronaut photography are an important and unique remote sensing method when monitoring natural disasters, urban growth, and environmental changes. While astronaut imagery can be used for earth science research, there is also an artistic aspect to the photography that fascinates a wide global population. A broader public audience can be introduced to earth science through high resolution, Earth art photos taken from the perspective of an astronaut.

The Crew Earth Observations (CEO) Facility within the Earth Science and Remote Sensing Unit at NASA's Johnson Space Center supports the acquisition, analysis, and curation of astronaut photography of Earth's surface and atmosphere. CEO's website, the Gateway to Astronaut Photography of Earth (eol.jsc.nasa.gov), provides free public access to view, search, and download over three million images taken by astronauts throughout all of NASA's crewed spaceflight history, with an emphasis on current ISS imagery. The CEO Facility actively curates a digital collection of exceptional Earth art astronaut photos used for public engagement. Our new Downloadable Earth Art page focuses on broad earth science topics including: mountains, water, clouds, agriculture, as well as an "abstract" category. This continuously-updated collection is comprised of freely accessible and high-quality downloadable materials, such as single- and dual-screen digital wallpapers. All Earth Art materials are presented with science-based information that complements the artistic qualities of the imagery, and facilitate connections between general audiences and earth science from the International Space Station. 